\title{
A PHYSIOTHERAPY PROGRAM FOR RECOVERY OF THE ANKLE/FOOT COMPLEX AFTER SURGERY TO CORRECT A CARTILAGE DEFECT (OSTEOCHONDRITIS DISSECANS) FOR IMPLEMENTATION IN THE PERIOD OF HOME RECOVERY
}

\author{
D. Vassileva-Decheva ${ }^{1}$ and Cv. Sokolov ${ }^{2}$ \\ ${ }^{1}$ University of Rousse "Angel Kanchev", Faculty of Public Health and Health Care, Department of Public \\ Health and Social Activity, Specialty Physiotherapy \\ 2University Hospital of Rousse, Department of "Orthopedics and Traumatology"
}

\begin{abstract}
The aim of this study is to build a suitable physiotherapy program for recovery of patients undergoing surgery in combination with platelet-rich plasma (PRP) therapy of the ankle joint, intended for implementation in the period of home treatment. This delineation of physiotherapy program contains an aim, the tasks set for realization of the aim and the used physiotherapy means. It is developed for the period of home treatment from postoperative week 2 (days 8-15) to the postoperative week 8 (days 56-63), the period that is very important for the normal recovery of the ankle/foot complex. The physiotherapy program is arranged into weeks and each of them contains the used physiotherapy means, the exercise duration and the therapeutic effect.
\end{abstract}

Key words: ankle/foot complex, talus cartilage defect, platelet-rich plasma (PRP), physiotherapy program for recovery, home treatment

Corresponding author: Denitza Vassileva-Decheva, PhD, University of Rousse "Angel Kanchev", Faculty of Public Health and Health Care, Department of Public Health and Social Activity, Section Physiotherapy, e-mail: ddecheva@uni-ruse.bg

\section{INTRODUCTION}

T he ankle/foot complex is one of the main structures of the bones and joints, which ensure the ability to perform a normal locomotor process. It is built of the talocruralis, subtalar, and transverse joints, as talus takes part in all of them so that $70 \%$ of the surface area is covered with cartilage. As a result, its state has a key role in carrying out the normal ankle/foot complex arthrokinematics [1]. Any defect in density, structure and smooth sliding in the ankle joint has a negative impact on the normal capacity for locomotion [4]. Surgical treatment and application of platelet-rich plasma (PRP) in the ankle/foot complex provides an opportunity for reli- able and quality recovery, but the requirement for a relatively long period of keeping non-attached structure leads to stagnation processes provoking painful symptoms, limitation of the normal volume of movement and significant muscle hypotrophy in the entire lower limb [3]. This determines the importance of the application of physical therapy during the postoperative period until full functional recovery of the ankle joint [2]. Due to the innovation of the method and the relatively recent use of platelet-rich plasma, in the scientific literature there is quite sparse data on the use of physical therapy after treatment with the method of platelet-rich plasma of the ankle joint. That prompted the need to build a complex of physiotherapy for recovery at home background. 


\section{THE AIM OF THE STUDY}

The aim of this study is to build a suitable physiotherapy program for recovery of patients undergoing surgery in combination with platelet-rich plasma therapy of the ankle joint, intended for implementation in the period of home treatment.

\section{MATERIAL AND METHODS}

This study is conducted in the period January - September 2016. Included patients are 48 - all of them operated in University Hospital of Rousse - in Department of Orthopedics and Traumatology with surgery to correct a cartilage defect on the ankle/foot complex in combination with platelet-rich plasma therapy. The patients were assigned into two groups (of 24 patients in each) - control and experimental. The difference between two groups was in the contents of period of home recovery. The patients included in the control group did not perform any physiotherapy program for home recovery. Those, who were included in the experimental group, performed a physiotherapy program for home implementation. The control group contained 10 men and 14 women at the average age of 31.5 years, the experimental -12 men and 12 women at the average age of 33 years.

The proposed physiotherapy program for treatment at home background is intended for patients who underwent surgery in the ankle/foot complex to remove cartilage defect and microfractures and received treatment by the method of platelet-rich plasma. The physiotherapy treatment in this type of treatment is divided into: 1. maximum-protective phase (conditionally covers the period from surgery until the end of the first postoperative month); 2. moderately-protective phase (conditionally it starts at the end of the first and conditionally until the end of the second postoperative month); 3. minimum-protective phase (conditionally it starts from the beginning of the third postoperative month until the end or until full functional recovery). Due to various individual characteristics, comorbidities or complications of treatment, the duration of the different phases can be changed. Developed physiotherapy treatment with home application covers a part of the maximum-protective and complete moderately-protective phase of recovery as patients usually are discharged for home treatment on the seventh postoperative day in the absence of post-operative complications. Imposed restrictions on loading and imposed full ability to relax ankle joint remain at the earliest by the end of the second postoperative month. Performance of the physiotherapy program can be carried out entirely by a physiotherapist or a previously trained relative of the patient under regular control of a physiotherapist.

The aim of this physiotherapy program is the functional recovery of the ankle/foot complex and preven- tion of potential complications of a hypostatic character.

The tasks set for the realization of the objective in this period are: psycho-emotional responses; resorption of postoperative edema; relief of pain syndrome; improvement of local blood supply - and lymphatic circulation; improvement of joint nutrition and healing; prevention of contractures; improvement of muscle - tendon elasticity; increase in muscle strength, increase of range of motion in the ankle joint and the entire lower limb due to the full ability to relax the limb, improving joint proprioception, strengthening the joint capsule - ligament apparatus, training in walking and overcoming obstacles with tools.

The used physiotherapy means are described in weeks. The implementation of the program starts immediately after the patient is discharged from the hospital to home care (in general on the seventh postoperative day - the end of the first postoperative week).

Table 1. Basic physiotherapy means used and their therapeutic influence in different structures in all period of physiotherapy home treatment

\begin{tabular}{|l|l|l|}
\hline Target system & Physiotherapy means & Therapeutic influence \\
\hline $\begin{array}{l}\text { Respiratory } \\
\text { system }\end{array}$ & Breathing exercises & $\begin{array}{l}\text { Hypostatic pneumonia } \\
\text { prophylaxis }\end{array}$ \\
\hline $\begin{array}{l}\text { Cardiovascular } \\
\text { system }\end{array}$ & Active motions & $\begin{array}{l}\text { Improvement of blood } \\
\text { circulation }\end{array}$ \\
\hline $\begin{array}{l}\text { Lymphatic and } \\
\text { blood system }\end{array}$ & Massage, ice bag & $\begin{array}{l}\text { Reduction of edemal } \\
\text { swelling, healing the pain }\end{array}$ \\
\hline Joint function & $\begin{array}{l}\text { Passive and active } \\
\text { motions }\end{array}$ & $\begin{array}{l}\text { Prevention of joint } \\
\text { contractures; Increase } \\
\text { of joint range of motion }\end{array}$ \\
\hline Muscle function & $\begin{array}{l}\text { Active motions, } \\
\text { isometric exercises, } \\
\text { resistance exercises, } \\
\text { PIR, stretching, recip- } \\
\text { rocal inhibition }\end{array}$ & $\begin{array}{l}\text { Improvement of tendon } \\
\text { - muscle elasticity, } \\
\text { increase of muscle } \\
\text { strength, normalization } \\
\text { of the muscle tonus }\end{array}$ \\
\hline Skin status & Anti-scar massage & $\begin{array}{l}\text { Prevention of dermal } \\
\text { and subdermal connec- } \\
\text { tive-tissue adhesions }\end{array}$ \\
\hline
\end{tabular}

Therefore, physiotherapy program composed for performance during the home treatment starts from the beginning of the second postoperative week and is as follows:

Postoperative week 2 (days 8-15): Physiotherapy procedure in this period begins with cryotherapy and massage by the method: a minute ice - a minute massage/a minute rubbing with an ice block in a wide area of the ankle joint (ventral, dorsal, medial and lateral), followed by a minute of surface, drainage mas- 
sage (total duration of 10 minutes). Since patients are still with surgical sutures and sterile bandage, protection against wetting is required. Then it is proceeded to the implementation of passive motions in the direction of dorsal and plantar flexion and implementation of circular motions in the ankle joint with duration of 5-8 minutes. In this period there are made also free active movements in the joint of the type „open kinetic chain" with recommended duration of 5-10 minutes or until provoking painful symptoms. For the prevention of muscle hypotrophy there are performed isometric contractions in series and for separate muscle groups, with the entire lower limb in the position of "Spitz" and "contra-Spitz". There are performed cryotherapy, massage, passive and active motions, and isometric contractions are made with starting position - supine lying. During this period, it is required to start training with walking aids - crutches or walker (depending on individual needs) and locomotion with overcoming obstacles - thresholds of doors or stairs. An important prerequisite in this stage of reconstruction is to facilitate the absorption of postoperative edema by positional treatment by lifting the distal leg - ankle at a higher level to the body.

Postoperative week 3 (days 16-23): During this period physiotherapy continues with the combined application of cryotherapy with massage by the same method (a minute ice - a minute massage). In this period the patients are with removed surgical sutures and without sterile dressing and this fact enables to expand the field of action. The massage is with surface drainage and anti-scar effects with prevention of dermal and subdermal connective-tissue adhesions. Duration of 10 minutes is maintained, and then it is passed to the embodiment of the passive, followed by free active motions in the ankle joint. It is continued with isometric contractions in series for the muscles of the entire lower limb. All the procedures continue to be carried out in starting supine position as it is recommended to increase the time for seating, standing and walking. At this stage positional treatment of the ankle joint continues. Movement is done with aids.

Postoperative week 4 (days 24-31): In the normal course of recovery and the lack of postoperative complications this period marks conditionally the end of the maximum-protective phase. Implementation of all physiotherapy means from the previous period of recovery continues, free active motions are performed by the sitting position to put drumsticks. At this stage, physiotherapy program is enriched by the active motions with appliances - „corn“, gymnastic ball and others. The duration is $10-15$ min or until provoking pain in the treated ankle joint. In this period it is recommended to put the plantar surface of the foot on uneven surfaces (prickly mat or corn cob) for recovery of plantar perception and improvement of joint proprioception. If there is still significant postoperative swelling in the ankle/foot complex, it is recommended continuation of positional therapy. Walking still takes place with crutches or a walker.

Postoperative week 5 (days 32-39): During this period, the moderately-protective phase of recovery usually begins. At this stage, the application of cryotherapy with massage described in the previous phase is still continued with free passive and active motions in supine position, but with reduced duration of 2-3 minutes. The onset of the moderately-protective phase includes exercises for mild to moderate resistance to manually force the muscles to perform the movements of the lower limb. This stage involves stretching of the Achilles tendon, postisometric relaxation and reciprocal inhibition at a moderate resistance to dorsal and plantar flexors, supinators and pronators (if needed) in the ankle joint. Positional treatment is applied only if there is a need of it. Due to restrictions on the burden on the treated ankle joint, the walking is still with aids.

Postoperative week 6 (days 40-47): Physiotherapy program in this period is based on the procedure from the previous phase and it is continued application of cryotherapy in combination with massage, with significantly reduced duration of the passive and free active motions to 1-2 min with retained duration 10-15 min of active motions with application of PIR and reciprocal inhibition, and muscle - tendon stretching. Exercises to increase muscle strength are performed manually against the resistance and isometric contractions are substituted by the exercises against elastic resistance (Thera band) for all major muscle groups, performing movements in the lower limb. Their duration continues for 10-15 repetitions for each muscle group and the starting positions are mainly sitting and standing (resting on the healthy leg). Locomotion continues to be carried out with aids.

Postoperative week 7 (days 48-55): At this stage, physiotherapy program is the same as that of the sixth postoperative week, with increasing the duration of exercises against elastic resistance to 20-30 repetitions for each exercised muscle group - the dorsal and plantar flexors, pronators and supinators muscles in the ankle joint, flexors and extensors in knee, flexors, extensors, abductors, and hip adductors. In this phase a partial removal of restrictions on the load on the treated ankle joint in the absence of complications starts, and only after the permission of the physician - orthopedist a gradual load on the joint begins and at the end of the period the patient is trained to walk again with an aid - a crutch.

Postoperative week 8 (days 56-63): This period marks the end of a moderately-protective phase of rehabilitation of patients with surgical treatment for 
cartilage defects and platelet-rich plasma application in the ankle/foot complex. If there is still a postoperative edema the application of the combined method of cryotherapy with massage can be extended. Passive and active free motions are applied only as needed. The emphasis is on the exercises, which duration is $15-20 \mathrm{~min}$. In the function of exercises for muscle strength, the exercises using an elastic resistance band are continued, as the duration increases to 30-40 repetitions for each muscle group exercised. If necessary, PIR, reciprocal inhibition and stretching are applied. At the end of the period, the patient is expected to walk without aids.

\section{RESULTS}

The outcomes of patients from the control and experimental groups were measured in the beginning (on postoperative day 8) and in the end (postoperative day 63 ) of their physiotherapy recovery period at home. The measured indices were anthropometric measurement of circumference of lower limb, range of motion in ankle, pain syndrome, muscle strength.

The outcomes of anthropometry measures (circumferences of shank, ankle joint, foot) show the next development in control and experimental groups with P-Value: 0,001.

Another observed index was the range of motions in ankle joint of patients into the two groups. The most important and significant of them, which were observed, were the dorsal and plantar flexions of talocrural joint. Results in the next figure show the development in this index in the beginning and in the end of physiotherapy recovery period of the patients in the control and experimental groups with P-Value: 0,005.

The index of pain is measured with the standard Visual Analogue Scale (VAS) in two states - motion and rest. Results show progressive reduction of the pain from the beginning to the end of patients recovery period, which is significant in patients from the experimental group in both testing states. The results are statistically significant with P-Value of 0.001 .

With standard Manual Muscle Testing there were measured the basic muscle groups, which provide the movement of ankle joint in sagittal plane. Essential differences were reported in strength of the plantar and dorsal flexor muscle groups in patients, including in experimental group. The results are shown in Table 3.The representative outcomes are in percentages (\%) and with P-Value: 0.000.

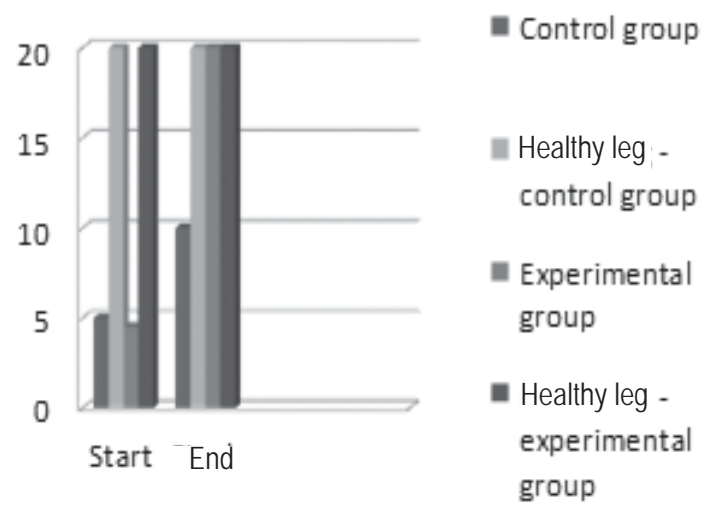

Fig. 1. Outcomes on dorsal flexion in the ankle joint (control and experimental groups)

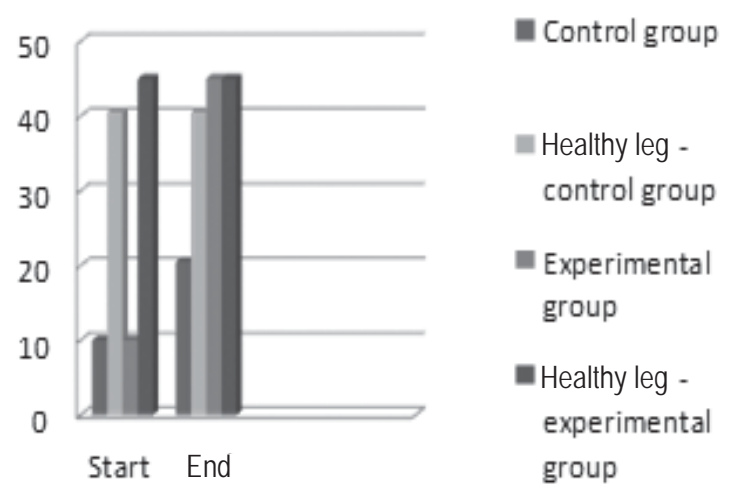

Fig. 2. Outcomes on plantar flexion in the ankle joint (control and experimental groups)

\section{VAS}

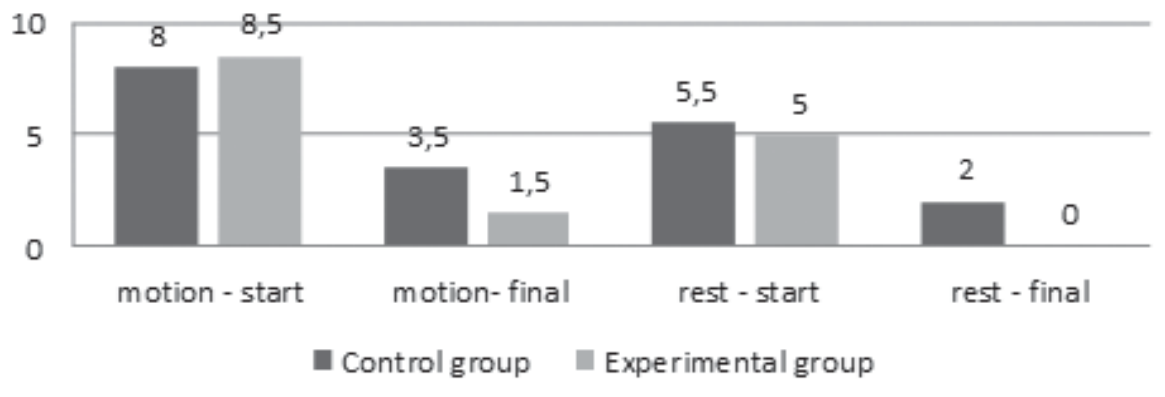

Fig. 3. Outcomes of VAS measurement in the patients of the two groups

\section{DISCUSSION}

The results obtained by measuring the circumference of the shank showed differences in the initial and final outcomes and those of the healthy leg only in the control group of patients. In this group under the same initial outcomes with those of the healthy $\operatorname{limb}-43 \mathrm{~cm}$, in the final outcome decrease of $1 \mathrm{~cm}$ is registered. It is suggested that this difference is due to hypotrophy of muscles of the shank, a result of non-destructive regimen of the lower limb and restriction of burden. 
Table 2. Average outcomes of leg circumferences (in centimeters $-\mathrm{cm}$ ) in patients of the two groups

\begin{tabular}{|l|c|c|c|c|c|c|}
\hline \multirow{3}{*}{ Index } & \multicolumn{4}{|c|}{ Operated leg } & \multicolumn{2}{c|}{ Healthy leg } \\
\cline { 2 - 7 } & \multicolumn{2}{|c|}{ Control group } & \multicolumn{2}{c|}{ Experimental group } & Control group & Experimental group \\
\cline { 2 - 7 } & Start & End & Start & End & Start & End \\
\hline Shank & 43 & 42 & 41.5 & 41.5 & 43 & 41.5 \\
\hline Ankle joint & 38.5 & 37.5 & 36 & 34.5 & 35.5 & 34.5 \\
\hline Foot & 32.5 & 33 & 31.5 & 30 & 31.5 & 30 \\
\hline
\end{tabular}

Table 3. Manual muscle strength outcomes in percentages

\begin{tabular}{|l|c|c|c|c|}
\hline \multirow{2}{*}{$\begin{array}{l}\text { Muscle } \\
\text { groups }\end{array}$} & \multicolumn{2}{|c|}{ Control group } & \multicolumn{2}{c|}{ Experimental group } \\
\cline { 2 - 5 } & Start & End & Start & End \\
\hline Plantar flexors & $32.80 \%$ & $62.70 \%$ & $35.40 \%$ & $88.50 \%$ \\
\hline Dorsal flexors & $28.60 \%$ & $53.50 \%$ & $30.10 \%$ & $89.60 \%$ \\
\hline
\end{tabular}

With regard to the outcomes registered in measuring the circumference of the ankle joint and foot there are found differences in both groups. In the control group outcomes differ by $2 \mathrm{~cm}$ at the ankle joint and with 1.5 $\mathrm{cm}$ in the foot compared to that of the healthy leg. This difference is still evident with existing edema in the lower limb at the end of postoperative day 63. In the experimental group there were observed high average outcomes at the beginning of period $-1.5 \mathrm{~cm}$, which contrasted with the healthy leg and disappeared during follow-up period (Table 2). The conclusion that can be made is that the reduction of postoperative edema is due to the physiotherapy resources, included in the program for home recovery.

As regards the amount of movement in the ankle joint in the direction of the plantar and dorsal flexions at approximately equal initial values in the two groups, there were observed better end results in the experimental one. On postoperative day 63, it was reported a significant increase in the range of motion in the ankle joint in patients from the experimental group that reaches that of the healthy leg - values of 45 degrees for plantar and 20 degrees of dorsal flexion. At the end of their recovery, the patients in the control group reached only half of the possible range of motion in the healthy leg (Fig. 1-2). Better results in the experimental group than the control group can be attributed to the effect of physiotherapy procedures included in the physiotherapy program for home recovery.

In assessing the sensation of pain again there were found better outcomes in the experimental group in motion and rest, with similar initial outcomes between the two groups (Fig. 3). These data allow us to conclude that physiotherapeutic methodology, developed for home use, helps reduce pain syndrome, both at rest and in motion.

The outcomes of the recovery of the muscle strength again are better in experimental group than the control group. It is obvious that with the similar initial outcomes between two groups, the final results are higher in the patients from the experimental group. In the control group final outcomes of the muscle strength of plantar flexors are $62.7 \%$ and for dorsal $-33.50 \%$, while in the experimental group the muscle strength of plantar flexors is $88.5 \%$ and for dorsal $-89.6 \%$ (Table 3 ). This significant difference in outcomes between the two groups proves the effectiveness of the proposed physiotherapy program for home implementation in regard to recovery of muscle strength, performing basic movement in the ankle joint.

\section{CONCLUSION}

Stagnant components of home care and the lack of adequate health competencies of the patients entail a significant risk of complications occurring as a result of hypokinesia that would aggravate their overall health and would stretch out the recovery period after surgery. By introducing physiotherapy program for home treatment that risk could be significantly minimized. The implementation of adequate and suitable rehabilitation program at home gives a chance for timely and quality recovery of patients undergoing surgery for a cartilage defect correction, combined with platelet-rich plasma application.

\section{REFERENCES}

1. Попов Н. Кинезиология и патокинезиология на опорнодвигателния апарат, НСА - Прес, София, 2009, 311.

2. Guney Ah., M. Akar, I. Karaman et al. Clinical outcomes of platelet-rich plasma (PRP) as an adjunct to microfracture surgery in osteochondral lesions of the talus; Knee surgery; Sports traumatology, Arthroscopy, August 2015, Volume 23, Issue 8, pp: 2384-2389.

3. Levangie P., C. Norkin. Joint Structure and Function, fifth edition; A Comprehensive Analysis; F.A Davis Company, 2011, 440.

4. Looze Ch., J. Capo, M. Ryan. Evaluation and Management of Osteochondral lesions of the Talus; SAGE JOURNAL, Cartilage, September 2016. 